\title{
Where are they? The inclusion of African-American men in empirical studies of type 2 diabetes self-care management
}

\author{
Ledric D. Sherman, E. Lisako J. McKyer \\ Department of Health and Kinesiology, Division of Health Education, College of Education and Human Development, Texas A \& M University, \\ College Station, Texas, United States
}

\section{A B S T R A C T}

Background:African-American Men experience higher rates of serious complications of diabetes, due in part to poor disease management. Yet it is unclear to what extent research been devoted to type 2 diabetes management in this population. Purpose: A need exists to clarify the extant literature on self-care management (SCM) practices of African-American Men with type 2 diabetes. Materials and Methods: A systematic literature and methodological quality scoring (MQS) using the Garrard matrix method was performed. Low scores (0-I) indicate low representation of African-American men; highest score (3) indicate high inclusion of African-American men in the samples. The search targeted articles focused on type 2 diabetes self-care management, and which included samples of African-American Men. Each publication was reviewed and assigned a MQS by the researchers, who reached 100\% concordance with the MQS. Results: Initial screening yielded I 22 articles, but only 4 I met full study inclusion criteria. These studies represent a combined sample size of $9,17 \mathrm{I}$ participants of which less than one-third (3,007; 32.8\%) were clearly identifiable as African-American men. Only 7 studies had samples consisting $100 \%$ of African-American Men. Mixed methods approaches were used least ( $n=9$ studies), followed by quantitative approaches ( $n=15$ studies). Qualitative approaches was most commonly used $(n=17$ studies). Most $(n=24)$ studies scored low $(0$ to I score), indicating low-level of inclusion of African-American Men in their sample. Discussion: In spite of the growing body of literature on managing type 2 diabetes, there is a paucity of information focused on a high-need and high-risk group - African-American Men. The exclusion of this population can result in adverse health consequences, given the high comorbidities associated with uncontrolled diabetes. Conclusion: Including more African-American Men in self-care management studies can help determine the factors affecting research participation among this group as well as to further understand the complexity that these men face regarding managing their diabetes.

Key words: Diabetes, African-American Men, Management

\section{INTRODUCTION}

Recruiting research participants in type 2 diabetes management research has proved to be a strenuous undertaking. ${ }^{[1]}$ The matter of recruiting is amplified when considering minority populations, specifically African-

\begin{tabular}{|l|l|}
\hline \multicolumn{2}{|c|}{ Access this article online } \\
\hline Quick Response Code: & Website: \\
\hline & www.josh.net \\
\hline & \\
\hline
\end{tabular}

American men. The limited inclusion of African-American men in type 2 diabetes self-care management (SCM) studies raises concern regarding how credible results from previous SCM studies can be generalized, as well as how valuable they can be for African-American men. Scarcely any studies have examined the beliefs and attitudes of African-American men, as well as African-Americans in general, with reference to anticipated impediments to being included and participating in research.

Several reasons are noted in the literature to explain low participation of racial/ethnic minorities in healthrelated research, including socioeconomic constraints, ${ }^{[2-6]}$ language and literacy barriers, ${ }^{[7,8]}$ lack of access to medical care, ${ }^{[4,9-11]}$ and the inability to recruit minorities into

Corresponding Author: Dr. Ledric Sherman, 4243 Texas A \& M University, College Station, Texas - 77843-4226, United States. E-mail: Isherman@hlkn.tamu.edu 
research studies. ${ }^{[12,13]}$ Mistrust of the scientific community is also theorized as a significant reason for the shortage of ethnic minorities in clinical studies. ${ }^{[7,14-24]}$

From a historical standpoint, the legacy of the enslavement of African-Americans sets a powerful basis for mistrust of authority figures and government leaders. ${ }^{[1,18,24]}$ In addition, the prominent Tuskegee syphilis experiment is an everpresent and painful reminder of African-American men's involvement in health-related research. The Tuskegee syphilis experiment study alone, has contributed to the construction of an immensely negative view of research and of healthcare professionals among African-Americans.

Previous studies of disparities in healthcare regarding race have documented patterns suggestive of African-American men's high levels of disengagement from healthcare organizations. ${ }^{[25-29]}$ African-American men generally attend fewer annual healthcare appointments than European American men ${ }^{[29]}$ and are less likely than African-American women to seek help from physicians. ${ }^{[30]}$ Accordingly, it is critical that African-American men with chronic diseases such as type 2 diabetes are able to successfully manage their conditions, especially if they are not likely to seek professional assistance.

\section{Diabetes SCM and African-American men}

African-American men experience higher rates of at least three serious complications of diabetes: Blindness, amputations, and end-stage renal disease (ESRD) compared to other groups. ${ }^{[30]}$ Despite the irregular burden of diabetes and its associated ramifications among African-American men, it is unclear to what extent any clinical or ethnographic research been devoted specifically to type 2 diabetes management in this population. For this reason, a critical need exists to improve what is known about the SCM practices of African-American men with type 2 diabetes.

The purpose of this systematic review is to identify and synthesize the research literature centered on one major research question: How well African-American men are included in empirical studies of diabetes SCM?

\section{MAterials And Methods}

The review process involved rigorous methodological initiatives to generate a comprehensive analysis of the published research literature on type 2 diabetes SCM. The methodology used for this systematic review is detailed below. Utilizing Garrard's matrix method ${ }^{[31]}$ of conducting systematic reviews, the following major steps were conducted:

1. Database search to identify relevant articles,

2. development of inclusion/exclusion criteria to select articles,
3. three-step screening process to identify SCM factors among published articles,

4. instrumentation to guide extraction process, and

5. data extraction to retrieve study characteristics among retrieved articles.

\section{Database search}

A systematic search was performed (per Garrard method) to retrieve peer-reviewed articles addressing SCM among AfricanAmerican men living with type 2 diabetes. Five major health literature databases: Academic Search Complete (EBSCO), ERIC (EBSCO), ScienceDirect (Elsevier), MEDLINE (Ovid), and PsycINFO were searched using keywords such as type 2 diabetes management, SCM, African-American men and type 2 diabetes, and men's health and type 2 diabetes. The date of the last search was August 2012.

\section{Inclusion/exclusion criteria}

Articles were selected if they (a) were empirical studies that included any reference to African-American men in their sample, (b) the published studies included sample participants with a medical diagnosis of type 2 diabetes, and (c) the publications were written and published in English between the years 1996 and 2012. The starting point (1996) was selected as it marks the availability and entree of diabetes blood testing strips as reflected in the research literature. All study design types were included (cross-sectional, focus groups, case-control, qualitative, quantitative, longitudinal, group randomized, and quasi-experimental). Exclusion criteria included (a) theoretical studies and thought pieces that did not included African-American men living with type 2 diabetes and (b) studies that did not address male involvement and participant in type 2 diabetes research.

\section{Screening of articles}

Screening process involved three tiers. First, screening questions based on inclusion and exclusion criteria were generated to guide retrieval, yielding 122 abstracts [Figure 1]. Second, full articles were evaluated for fit with

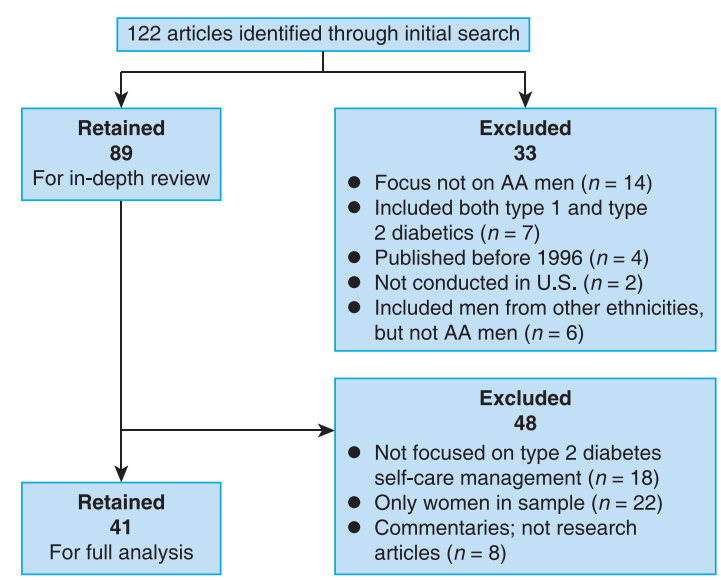

Figure 1: 122 articles identified through initial search 
other inclusion criteria. Irrelevant titles, duplicates, and narrative/commentary pieces were automatically excluded. Studies that addressed type 2 diabetes SCM were retained. Third, additional articles were identified by purling, that is, the performance of a thorough review of the references/ citations of retrieved articles for publications that might have been missed through the database search. Purling is often performed to ensure that all relevant articles are retrieved (Garrard, 2004).

\section{Instrumentation}

A methodological quality scoring (MQS) rubric ${ }^{[31]}$ was tailored to the needs of our study and was used to standardize data extraction methods applied to reviewed studies. The MQS was designed to guide identification and assessment of methodological characteristics among reviewed articles (e. g., key factors associated with SCM, characteristics of the measures used to assess such factors). The MQS also details the data extraction process, the type of information extracted from reviewed studies, and the rationale used to determine key study and methodological characteristics. Specific to this study, we sought to identify and qualify the extent to which African-American men were included in SCM studies. The scoring criteria are described in Table 1.

\section{Data extraction}

Characteristics of articles (e. g., purpose of study, study design, and theoretical framework) were entered into the MQS, and then categorized based on similarity of study aims, and of investigated factors (e. g., health literacy and its association with diabetes knowledge, perceived selfefficacy, and disease self-management).

\section{RESULTS}

The research question guiding the study focused on the extent to which African-American men are included in empirical studies of diabetes SCM.

\begin{tabular}{lrl}
\hline Table 1: Scoring rubric for research articles \\
\hline $\begin{array}{l}\text { African-American (AA) } \\
\text { men targeted and included }\end{array}$ & $\begin{array}{l}\text { Studies focused only on AA men with } \\
\text { 100\% AA men in sample } \\
\text { Studies with AA men and women, with } \\
\text { at least 50\% of their sample are AA men }\end{array}$ \\
$\begin{array}{l}\text { AA men targeted, some } \\
\text { included }\end{array}$ & $\begin{array}{l}\text { Studies focused on AA men and women } \\
\text { and we can determine \% of AA men in } \\
\text { their sample }\end{array}$ \\
$\begin{array}{l}\text { AA men targeted; none } \\
\text { included or cannot } \\
\text { determine }\end{array}$ & $\begin{array}{l}\text { AA men appear to be targeted, but } \\
\text { none included or insufficient info to } \\
\text { AA men neithertargetednor } \\
\text { included or cannot } \\
\text { determine }\end{array}$ & $\begin{array}{l}\text { Nolate \% information is available in the } \\
\text { methods section about whether or not } \\
\text { they tried to include AA men }\end{array}$ \\
\hline
\end{tabular}

\section{Description of studies}

Among 122 articles initially identified as publications highlighting research that has achieved inclusion of African-American men in type 2 diabetes self-management related studies, $41(33.6 \%)$ met the final criteria. The combined sample across these studies represent 9,171 participants, of which $3,924(42.72 \%)$ are men of any race/ethnicity and 3,007 (32.8\%) were clearly identified as African-American men. Among these, seven studies (17.1\% of studies) (combined $N=1,350 ; 14.7 \%$ of combined samples) focused exclusively on African-American men. Seven other publications included samples described such that participants' sex or racial/ethnic characteristics could not be disaggregated. Consequently, for these studies representing 2,328 participants or $25.4 \%$ of participants across all studies, the numbers and proportion of AfricanAmerican men could not be calculated. Table 2 provides full details of our findings.

\section{Analysis features}

Seven studies received the lowest score of "0" [Table 2], indicating the lack of data needed to determine the extent to which African-American men were included. A similar number of studies achieved the highest score of 3 , denoting efforts to exclusively target African-American men for their sample and achieving that aim. Most $(n=24 ; 58.5 \%$ of studies) received a "1" inclusionscore, indicating that information was available for us to i) ascertain that African-American men were part of those targeted in some form, and 2) calculate the proportion of African-American men actually included in the study. As per Table 2, African-American men were included at an average of about 30\% among studies with "1" score.

\section{Methodological approaches utilized in research}

Qualitative approaches accounted for most of the studies ( $n=17 ; 41.5 \%$ of studies), followed by qualitative $(n=15$; $36.6 \%$ of studies $)$ and mixed methods $(n=9 ; 22.0 \%$ of studies). All three types had more than half of their respective studies score low (i. e., 1) [Table 1]. Yet studies which utilized mix methods had a larger proportion which scored low (44.4\% with '0' score, and 55.6\% with " 1 " score) relative to studies using other methods. None of the mixed methods studies received a score higher than 1 .

Among the seven studies exclusively focused on AfricanAmerican men, four used qualitative approaches and none were mixed methods. The three quantitative studies were performed by the same lead author and their team.

\section{DISCUSSION}

Insufficient attention has been centered on the recruitment and retention of African-American men in research, as well 


\begin{tabular}{|c|c|c|c|c|c|c|c|c|c|c|c|c|c|c|c|c|c|c|}
\hline Authors & Year & Approach & Sample & Total & Men & Women & $\begin{array}{l}\text { AA men/ } \\
\text { women }\end{array}$ & $\begin{array}{l}\text { "AA } \\
\text { men }\end{array}$ & $\begin{array}{l}\% \\
\text { men }\end{array}$ & $\begin{array}{l}\% \text { AA } \\
\text { men }\end{array}$ & Score & & & & $\begin{array}{l}\text { Theoretical } \\
\text { framework }\end{array}$ & & $\begin{array}{l}\text { Data collection } \\
\text { method }\end{array}$ & Key findings \\
\hline $\begin{array}{l}\text { Anderson } \\
\text { et al. }\end{array}$ & 1996 & Qualitative & $\begin{array}{l}\text { AA men } \\
\text { and } \\
\text { women } \\
\text { with } \\
\text { T2DM }\end{array}$ & 34 & 12 & 22 & 34 & 12 & 35.3 & 35.3 & 3 & & & US & Not reported & 3 & $\begin{array}{l}\text { Focus group } \\
\text { questions }\end{array}$ & $\begin{array}{l}\text { Identify issues that } \\
\text { could serve as topics for } \\
\text { a series of educational } \\
\text { videos portraying } \\
\text { psychosocial issues of } \\
\text { urban black individuals } \\
\text { with diabetes }\end{array}$ \\
\hline $\begin{array}{l}\text { El-Kebbi } \\
\text { et al. }\end{array}$ & 1996 & Qualitative & $\begin{array}{l}\text { AA } \\
\text { adults } \\
\text { with } \\
\text { T2DM }\end{array}$ & 45 & - & - & 45 & - & - & $\begin{array}{l}\text { Undete- } \\
\text { rmined }\end{array}$ & 3 & 1,322 & AA adults & US & Not reported & 3 & $\begin{array}{l}\text { Open-ended } \\
\text { interview } \\
\text { question guide }\end{array}$ & $\begin{array}{l}\text { Potential barriers that } \\
\text { too dietary adherence } \\
\text { among low-income, } \\
\text { urban black patients } \\
\text { with T2DM }\end{array}$ \\
\hline $\begin{array}{l}\text { Resnick } \\
\text { et al. }\end{array}$ & 1998 & Quantitative & $\begin{array}{l}\text { AA men } \\
\text { and } \\
\text { women } \\
\text { with } \\
\text { T2DM }\end{array}$ & 1,531 & 591 & 940 & 1,531 & 591 & 38.6 & 38.6 & 3 & "REF! & $\begin{array}{l}\text { Blacks } \\
\text { andwhites }\end{array}$ & US & Not reported & 3 & $\begin{array}{l}\text { Baseline } \\
\text { interview } \\
\text { questions } \\
\text { and medical } \\
\text { examination; } \\
\text { data from three } \\
\text { NHEFS follow- } \\
\text { up interviews } \\
\text { 1982-1984, } \\
\text { 1987, and } 1992\end{array}$ & $\begin{array}{l}\text { Associations of BMI } \\
\text { and fat distribution with } \\
\text { diabetes are modified } \\
\text { by race }\end{array}$ \\
\hline $\begin{array}{l}\text { Anderson- } \\
\text { Loftin and } \\
\text { Moneyham }\end{array}$ & 2000 & Qualitative & $\begin{array}{l}\text { AA } \\
\text { adults } \\
\text { with } \\
\text { T2DM }\end{array}$ & 22 & - & - & 22 & - & - & $\begin{array}{l}\text { Undete- } \\
\text { rmined }\end{array}$ & 3 & \#REF! & & US & $\begin{array}{l}\text { Nursing care } \\
\text { management } \\
\text { model (major } \\
\text { concepts: Sick } \\
\text { care, health- } \\
\text { making, } \\
\text { nurse-client } \\
\text { relationship) }\end{array}$ & 3 & $\begin{array}{l}\text { Questions } \\
\text { during the focus } \\
\text { group sessions }\end{array}$ & $\begin{array}{l}\text { Symptom management; } \\
\text { health choices; health } \\
\text { and social services; } \\
\text { characteristics of } \\
\text { healthcare providers }\end{array}$ \\
\hline $\begin{array}{l}\text { Fitzgerald } \\
\text { et al. }\end{array}$ & 2000 & Quantitative & $\begin{array}{l}\text { AA and } \\
\text { whites } \\
\text { with } \\
\text { T2DM }\end{array}$ & 672 & - & - & - & - & - & $\begin{array}{l}\text { Undete- } \\
\text { rmined }\end{array}$ & 1 & & & US & Not reported & 1 & $\begin{array}{l}\text { Diabetes care } \\
\text { profile (DCP) }\end{array}$ & $\begin{array}{l}\text { Attitudes toward } \\
\text { diabetes as measured } \\
\text { by the } 16 \text { scales of the } \\
\text { DCP differ by diabetes } \\
\text { treatment modality and } \\
\text { race/ethnicity }\end{array}$ \\
\hline $\begin{array}{l}\text { Hendricks } \\
\text { and } \\
\text { Hendricks }\end{array}$ & 2000 & Qualitative & $\begin{array}{l}\text { AA men } \\
\text { with } \\
\text { T2DM }\end{array}$ & 30 & 30 & NA & 30 & 30 & 100.0 & 100.0 & 3 & & & US & Not reported & 3 & $\begin{array}{l}\text { Educational } \\
\text { classes; follow- } \\
\text { up telephone } \\
\text { call }\end{array}$ & $\begin{array}{l}\text { A1c level; perception of } \\
\text { general health; present } \\
\text { diabetes knowledge; } \\
\text { daily foot care; dietary } \\
\text { patterns; exercise and } \\
\text { medication patterns }\end{array}$ \\
\hline Aljsem et al. & 2001 & Quantitative & $\begin{array}{l}\text { AA men } \\
\text { and } \\
\text { women } \\
\text { with } \\
\text { T2DM }\end{array}$ & 308 & 187 & 121 & 308 & 187 & 60.7 & 60.7 & 3 & & & US & $\begin{array}{l}\text { Health belief } \\
\text { model; } \\
\text { self-efficacy } \\
\text { concept }\end{array}$ & 1 & $\begin{array}{l}\text { Self-reported } \\
\text { questionnaires }\end{array}$ & $\begin{array}{l}\text { Relationships of } \\
\text { diabetes-specific } \\
\text { treatment barriers and } \\
\text { self-efficacy with self- } \\
\text { care behaviors }\end{array}$ \\
\hline
\end{tabular}




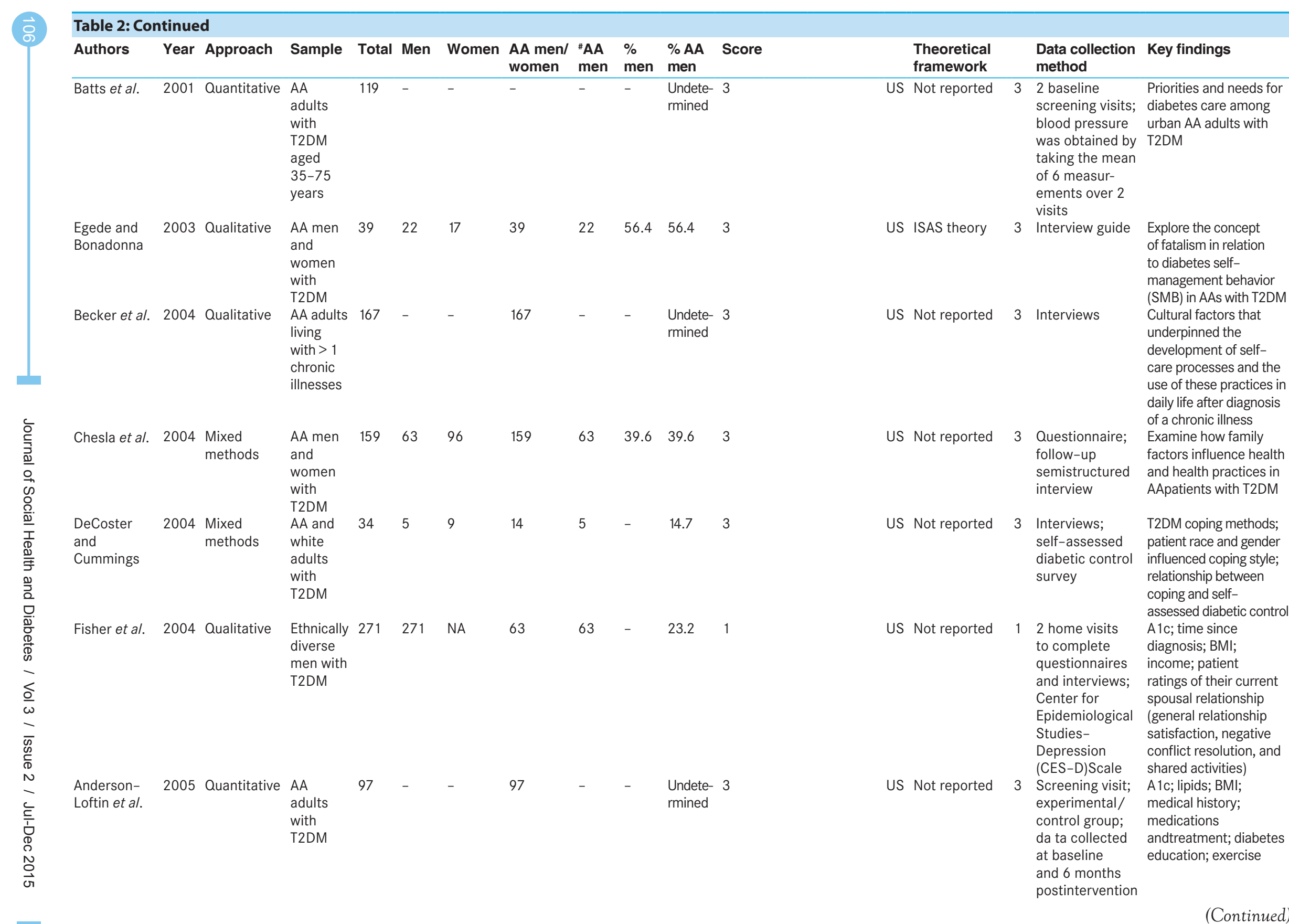




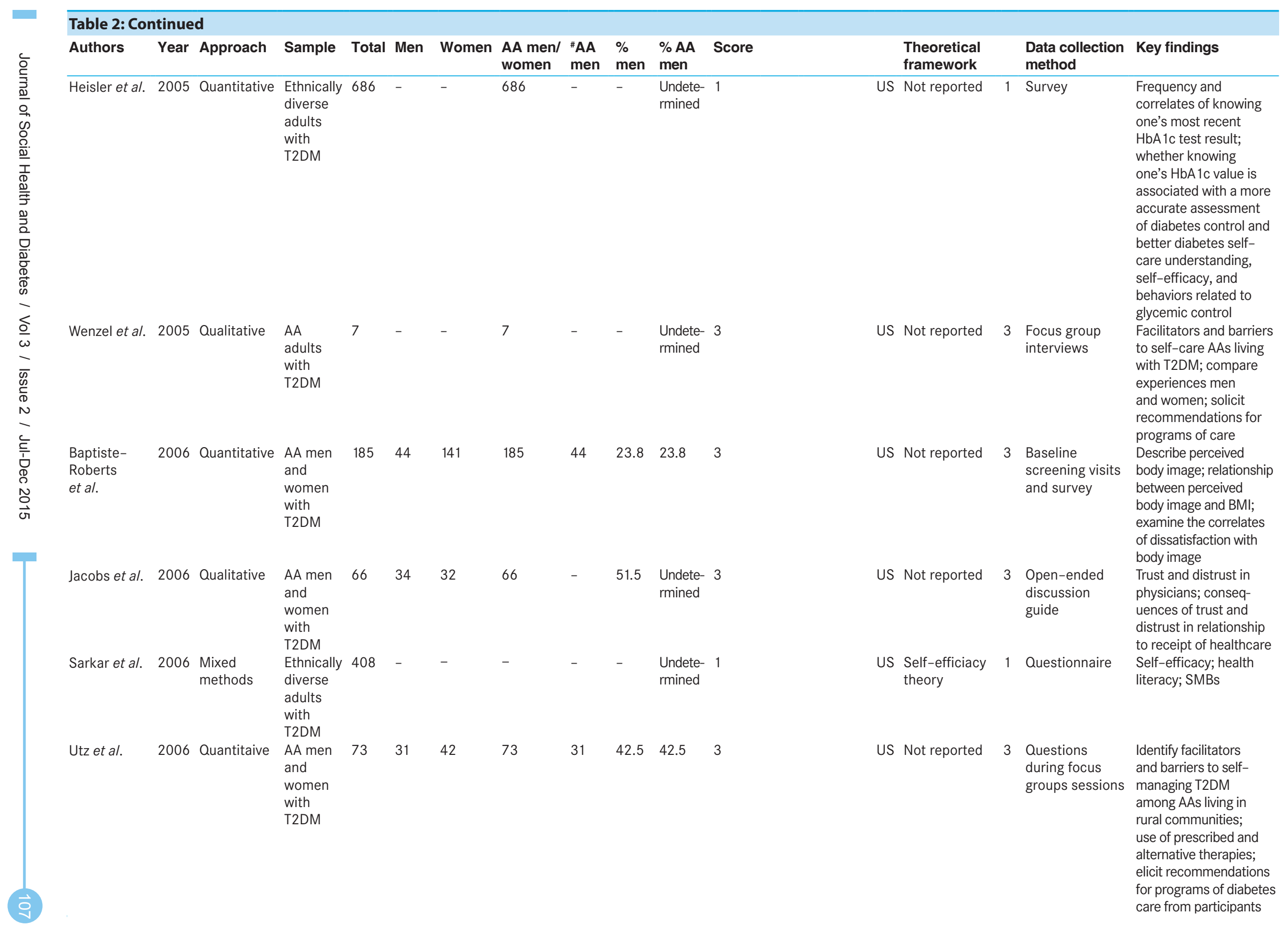




\begin{tabular}{|c|c|c|c|c|c|c|c|c|c|c|c|c|c|c|c|c|}
\hline \multicolumn{17}{|c|}{ Table 2: Continued } \\
\hline Authors & Year & Approach & Sample & Total & Men & Women & $\begin{array}{l}\text { AA men/ } \\
\text { women }\end{array}$ & $\begin{array}{l}{ }^{\# A A} \\
\text { men }\end{array}$ & $\begin{array}{l}\% \\
\text { men }\end{array}$ & $\begin{array}{l}\% \text { AA } \\
\text { men }\end{array}$ & Score & & $\begin{array}{l}\text { Theoretical } \\
\text { framework }\end{array}$ & & $\begin{array}{l}\text { Data collection } \\
\text { method }\end{array}$ & Key findings \\
\hline DeWalt et al. & 2007 & $\begin{array}{l}\text { Mixed } \\
\text { methods }\end{array}$ & $\begin{array}{l}\text { AA and } \\
\text { whites } \\
\text { with } \\
\text { T2DM }\end{array}$ & 268 & - & - & - & - & - & $\begin{array}{l}\text { Undete- } \\
\text { rmined }\end{array}$ & 1 & US & Not reported & 1 & Questionnaire & A1c; literacy; trust \\
\hline Liburd et al. & 2007 & Qualitative & $\begin{array}{l}\text { AA men } \\
\text { with } \\
\text { T2DM }\end{array}$ & 16 & 16 & NA & 16 & 16 & 100.0 & 100.0 & 3 & US & Not reported & 3 & Interviews & $\begin{array}{l}\text { Themes that emerged } \\
\text { in illness narratives of } \\
\text { a small sample of AA } \\
\text { men living with T2DM; } \\
\text { lived experience of } \\
\text { Black manhood and } \\
\text { masculinity and its } \\
\text { intersection with the } \\
\text { challenges of diabetes } \\
\text { self-management }\end{array}$ \\
\hline Polzer & 2007 & Qualitative & $\begin{array}{l}\text { AA men } \\
\text { and } \\
\text { women } \\
\text { with } \\
\text { T2DM }\end{array}$ & 29 & 10 & 19 & 29 & 10 & 34.5 & 34.5 & 3 & US & $\begin{array}{l}\text { Grounded } \\
\text { theory }\end{array}$ & 3 & Interviews & $\begin{array}{l}\text { Asking participants } \\
\text { what it was like for } \\
\text { them taking care of } \\
\text { their diabetes and } \\
\text { how their spritual } \\
\text { beliefs and practices } \\
\text { affected their self- } \\
\text { management }\end{array}$ \\
\hline $\begin{array}{l}\text { Polzer and } \\
\text { Miles }\end{array}$ & 2007 & Qualitative & $\begin{array}{l}\text { AA men } \\
\text { and } \\
\text { women } \\
\text { with } \\
\text { T2DM }\end{array}$ & 29 & 10 & 19 & 29 & 10 & 34.5 & 34.5 & 3 & US & $\begin{array}{l}\text { Grounded } \\
\text { theory }\end{array}$ & 3 & $\begin{array}{l}\text { Interview } \\
\text { questions }\end{array}$ & $\begin{array}{l}\text { How spirituality affects } \\
\text { self-management of } \\
\text { T2DM }\end{array}$ \\
\hline Jones et al. & 2008 & $\begin{array}{l}\text { Mixed } \\
\text { methods }\end{array}$ & $\begin{array}{l}\text { Rural } \\
\text { AAs with } \\
\text { T2DM }\end{array}$ & 21 & - & - & 21 & - & - & $\begin{array}{l}\text { Undete- } \\
\text { rmined }\end{array}$ & 3 & US & Not reported & 3 & $\begin{array}{l}\text { Group sessions } \\
\text { to obtain } \\
\text { information } \\
\text { about diabetes } \\
\text { and family/peer } \\
\text { support }\end{array}$ & $\begin{array}{l}\text { Impact of family } \\
\text { and friends on the } \\
\text { management of } \\
\text { persons with diabetes } \\
\text { and their willingness } \\
\text { to be involved in a } \\
\text { culturally tailored } \\
\text { program }\end{array}$ \\
\hline Peek et al. & 2008 & Qualitative & $\begin{array}{l}\text { AA } \\
\text { adults } \\
\text { with } \\
\text { T2DM }\end{array}$ & 48 & - & - & 48 & - & - & $\begin{array}{l}\text { Undete- } \\
\text { rmined }\end{array}$ & 3 & US & $\begin{array}{l}\text { Interview } \\
\text { guides } \\
\text { created based } \\
\text { on Theory } \\
\text { of Planned } \\
\text { Behavior, } \\
\text { Ecological } \\
\text { Model, and } \\
\text { Shared } \\
\text { Decision } \\
\text { Making Model }\end{array}$ & 3 & $\begin{array}{l}\text { Topic guides } \\
\text { for in-depth } \\
\text { interviews; } \\
\text { focus groups }\end{array}$ & $\begin{array}{l}\text { Patient definitions and } \\
\text { perceptions of shared } \\
\text { decision making; } \\
\text { barriers and facilitators } \\
\text { of SDM; perceived } \\
\text { impact of race/culture } \\
\text { on SDM }\end{array}$ \\
\hline
\end{tabular}




\begin{tabular}{|c|c|c|c|c|c|c|c|c|c|c|c|c|c|c|c|c|c|}
\hline \multirow{3}{*}{$\begin{array}{l}\text { Table 2: Co } \\
\text { Authors } \\
\text { Rosland } \\
\text { et al. }\end{array}$} & \multirow{3}{*}{$\begin{array}{r}\text { ntinue } \\
\text { Year } \\
2008\end{array}$} & \multirow{3}{*}{$\begin{array}{l}\text { Approach } \\
\text { Mixed } \\
\text { methods }\end{array}$} & \multirow{3}{*}{$\begin{array}{l}\text { Sample } \\
\text { AA } \\
\text { adults } \\
\text { with } \\
\text { T2DM }\end{array}$} & \multirow{3}{*}{$\begin{array}{l}\text { Total } \\
94\end{array}$} & \multirow[b]{2}{*}{ Men } & \multirow[b]{2}{*}{ Women } & \multirow[b]{2}{*}{$\begin{array}{l}\text { AA men/ } \\
\text { women }\end{array}$} & \multirow[b]{2}{*}{$\begin{array}{l}\text { "AA } \\
\text { men }\end{array}$} & \multirow[b]{2}{*}{$\begin{array}{l}\% \\
\text { men }\end{array}$} & \multirow[b]{2}{*}{$\begin{array}{l}\% \text { AA } \\
\text { men }\end{array}$} & \multirow[b]{2}{*}{ Score } & \multirow{2}{*}{\multicolumn{2}{|c|}{$\begin{array}{l}\text { Theoretical } \\
\text { framework }\end{array}$}} & \multirow{2}{*}{\multicolumn{2}{|c|}{$\begin{array}{l}\text { Data collection } \\
\text { method }\end{array}$}} & \multirow[b]{2}{*}{ Key findings } & \\
\hline & & & & & & & & & & & & & & & & & \\
\hline & & & & & - & - & 94 & - & - & $\begin{array}{l}\text { Undete- } \\
\text { rmined }\end{array}$ & 1 & US & Not reported & 1 & $\begin{array}{l}\text { Suverys } \\
\text { andquestionnaire }\end{array}$ & $\begin{array}{l}\text { Sociodemographics; } \\
\text { health status; A1c } \\
\text { levels; social support } \\
\text { from family and friends; } \\
\text { diabetes SMBs; physical } \\
\text { activity; diabetes care } \\
\text { self-efficacy,and } \\
\text { depressive symptoms }\end{array}$ & \\
\hline Tang et al. & 2008 & $\begin{array}{l}\text { Mixed } \\
\text { methods }\end{array}$ & $\begin{array}{l}\text { AA } \\
\text { adults } \\
\text { with } \\
\text { T2DM }\end{array}$ & 89 & - & - & 89 & - & - & $\begin{array}{l}\text { Undete- } \\
\text { rmined }\end{array}$ & 3 & US & $\begin{array}{l}\text { Symbolic } \\
\text { interaction } \\
\text { theory }\end{array}$ & 3 & Survey & $\begin{array}{l}\text { Diabetes-related social } \\
\text { support; medication/ } \\
\text { insulin use; foot care; } \\
\text { self-monitoring of } \\
\text { blood glucose; physical } \\
\text { activity (PA); dietary } \\
\text { patterns; diabetes } \\
\text { specific quality of life }\end{array}$ & $\frac{\mathscr{N}}{\widetilde{D}}$ \\
\hline Duru et al. & 2009 & Quantitative & $\begin{array}{l}\text { AA/ } \\
\text { blacks } \\
\text { and } \\
\text { whites } \\
\text { with } \\
\text { diabetes }\end{array}$ & 764 & - & - & 205 & - & - & $\begin{array}{l}\text { Undete- } \\
\text { rmined }\end{array}$ & 3 & US & Not reported & 3 & $\begin{array}{l}\text { Analysis used } \\
\text { data from a } \\
\text { Translating } \\
\text { Research } \\
\text { Into Action } \\
\text { for Diabetes } \\
\text { (TRIAD) } \\
\text { questionnaire }\end{array}$ & $\begin{array}{l}\text { Whether several risk } \\
\text { factors(Hb A1c; systolic } \\
\text { blood pressure; higher } \\
\text { low-density lipoprotein) } \\
\text { were more strongly } \\
\text { associated with poor } \\
\text { control of multiple } \\
\text { intermediate outcomes } \\
\text { among blacks with } \\
\text { diabetes than among } \\
\text { similar whites }\end{array}$ & 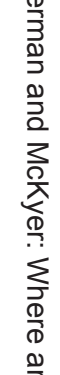 \\
\hline $\begin{array}{l}\text { Samuel- } \\
\text { Hodge et al. }\end{array}$ & 2009 & Quantitative & $\begin{array}{l}\text { AA } \\
\text { adults } \\
\text { with } \\
\text { T2DM }\end{array}$ & 117 & - & - & 117 & - & - & $\begin{array}{l}\text { Undete- } \\
\text { rmined }\end{array}$ & 3 & US & Not reported & 3 & $\begin{array}{l}\text { Physiologic } \\
\text { measures; PA } \\
\text { monitor; food } \\
\text { questionnaire; } \\
\text { diabetes } \\
\text { knowledge } \\
\text { scale }\end{array}$ & $\begin{array}{l}\text { A1c; body weight; blood } \\
\text { pressure; PA; food } \\
\text { frequency; diabetes } \\
\text { knowledge }\end{array}$ & 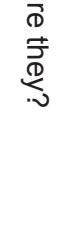 \\
\hline $\begin{array}{l}\text { Thompson } \\
\text { et al. }\end{array}$ & 2009 & Qualitative & $\begin{array}{l}\text { AA men } \\
\text { with } \\
\text { T2DM }\end{array}$ & 43 & 43 & 0 & 43 & 43 & 100.0 & 100.0 & 3 & US & Non reported & 3 & $\begin{array}{l}\text { Focus groups } \\
\text { interviews }\end{array}$ & $\begin{array}{l}\text { What healthinformation } \\
\text { and needs do AA } \\
\text { men have? How do } \\
\text { AA men describe } \\
\text { their efforts to obtain } \\
\text { health information? } \\
\text { What factors facilitate } \\
\text { or inhibit health- } \\
\text { informationseeking by } \\
\text { AA men? }\end{array}$ & \\
\hline $\begin{array}{l}\text { Chlebowy } \\
\text { et al. }\end{array}$ & 2010 & $\begin{array}{l}\text { Mixed } \\
\text { methods }\end{array}$ & $\begin{array}{l}\text { AA } \\
\text { adults } \\
\text { with } \\
\text { T2DM }\end{array}$ & 38 & - & - & 38 & - & - & $\begin{array}{l}\text { Undete- } \\
\text { rmined }\end{array}$ & 3 & US & Not reported & 3 & $\begin{array}{l}\text { Interview } \\
\text { questions in } \\
\text { focus group }\end{array}$ & $\begin{array}{l}\text { Facilitators and barriers } \\
\text { to self-management of } \\
\text { T2DM }\end{array}$ & \\
\hline
\end{tabular}




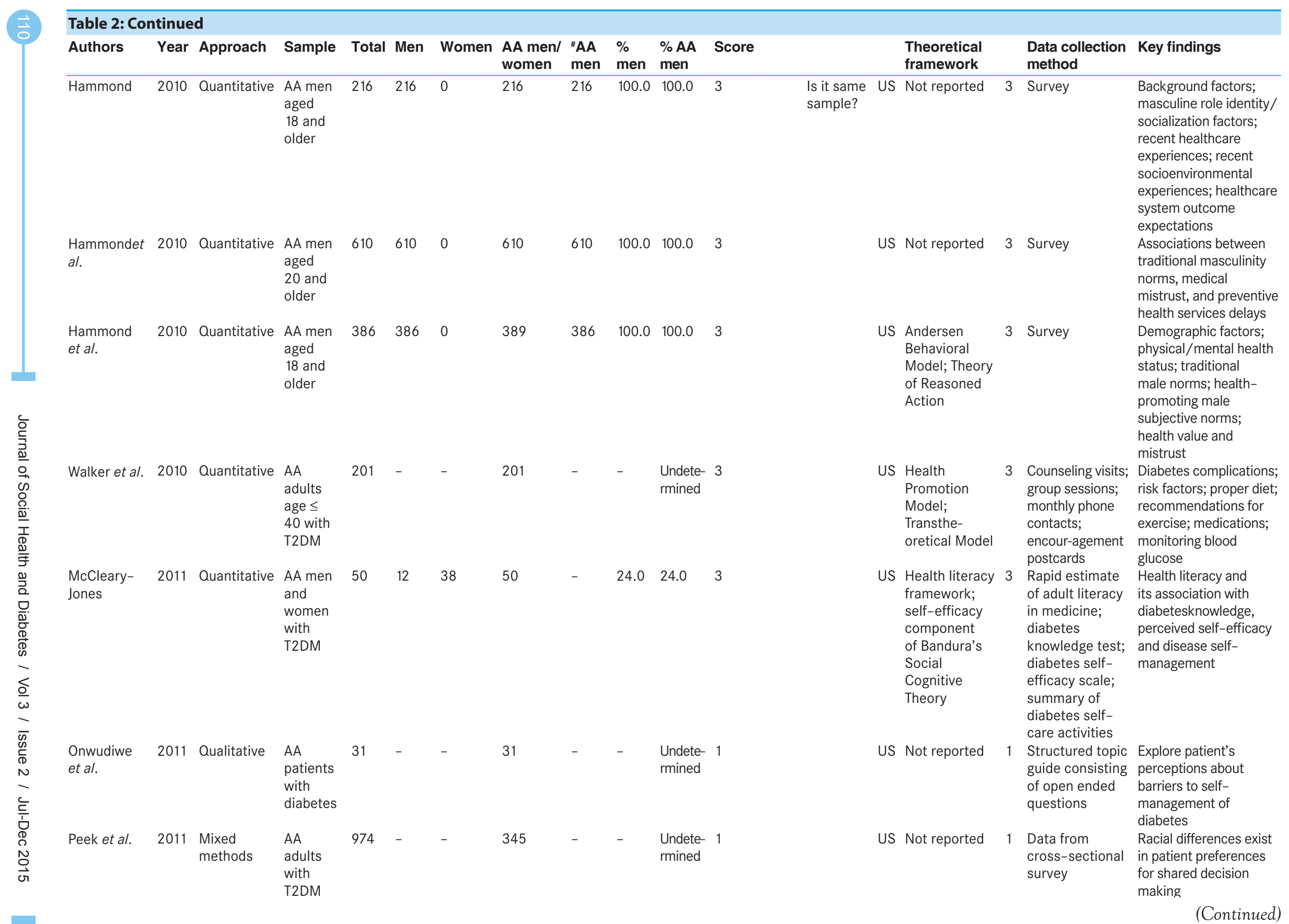




\begin{tabular}{|c|c|c|c|c|c|c|c|c|c|c|c|c|c|c|c|c|}
\hline Authors & Year & Approach & Sample & Total & Men & Women & $\begin{array}{l}\text { AA men/ } \\
\text { women }\end{array}$ & $\begin{array}{l}{ }^{\text {AAA }} \\
\text { men }\end{array}$ & $\begin{array}{l}\% \\
\text { men }\end{array}$ & $\begin{array}{l}\% \text { AA } \\
\text { men }\end{array}$ & Score & & $\begin{array}{l}\text { Theoretical } \\
\text { framework }\end{array}$ & & $\begin{array}{l}\text { Data collection } \\
\text { method }\end{array}$ & Key findings \\
\hline Bhattacharya & 2012 & Qualitative & $\begin{array}{l}\text { AA men } \\
\text { and } \\
\text { women } \\
\text { living with } \\
\text { T2DM }\end{array}$ & 31 & 15 & 16 & 31 & - & 48.4 & 48.4 & 3 & US & $\begin{array}{l}\text { Self- } \\
\text { Determination } \\
\text { Theory; } \\
\text { Grounded } \\
\text { Theory }\end{array}$ & 3 & Interview & $\begin{array}{l}\text { Psychosocial and } \\
\text { emotional issues on } \\
\text { being diagnosed with } \\
\text { T2DM. }\end{array}$ \\
\hline \multirow[t]{2}{*}{ Hooker et al. } & 2012 & Qualitative & $\begin{array}{l}\text { AA men } \\
\text { aged } \\
45-88\end{array}$ & 49 & 49 & - & 49 & - & 100.0 & 100.0 & 3 & US & Not reported & 3 & $\begin{array}{l}\text { One-on-one } \\
\text { interview }\end{array}$ & $\begin{array}{l}\text { Ascertain perspectives } \\
\text { on masculinity in AA } \\
\text { men aged } 45-84 \text { years; } \\
\text { determine if these } \\
\text { perspectives vary by age } \\
\text { or PA behavior; identify } \\
\text { the potential influence } \\
\text { these views may have } \\
\text { on health; distinguish } \\
\text { how these perspectives } \\
\text { may be applicable to } \\
\text { engaging older AA men in } \\
\text { community-based health } \\
\text { promotion }\end{array}$ \\
\hline & & $\begin{array}{l}\text { Total } \\
\text { sample size }\end{array}$ & AA men & 9,057 & 2,657 & 1,512 & 6,177 & 2,339 & 25.8 & & & & & & & \\
\hline \multirow{2}{*}{$\begin{array}{l}4 \text { qual and } \\
2 \text { quant } \\
\text { (Hammond) }\end{array}$} & & 1,350 & 1,350 & & & & & & & & & & & & & \\
\hline & & $\begin{array}{l}413 \\
\text { \#REF! }\end{array}$ & 243 & & & & & & & & & & & & & \\
\hline
\end{tabular}


as barriers to entry into studies, therefore resulting in the current gap in research literature. The objective of this study was to analyze the research literature in order to ascertain the extent to which African-American menare included in empirical studies of type 2 diabetes SCM. Our results reveal an abysmally low number of studies targeting African-American men, as well as a low number and proportion of African-American men comprising the limited number of studies.

More than half of the seven published studies exclusively focused on African-American men utilize qualitative approaches. While qualitative methods are not problematic, and indeed are critical for exploratory phases of understanding phenomenon, it also is an indicator of the slow progress being made toward understanding type-2 diabetes management issues specific to African-American men that have not yet gone beyond exploration.

Studies were found that included African-American men, but there were several for which we were unable to ascertain their actual numbers or proportion. This was due largely to the lack of details in the methods sections describing recruitment protocols and final samples, as well as in the results sections of these various papers. One reason for the lack of information may be that obtaining AfricanAmerican men in particular was not the aim, but rather the outcome of achieving a diverse sample. Other reasons may be word limits faced by authors, as they struggle to include critical information in a constrained amount of space. In either of these cases, it is understandable in such case why the details would not be included. Nevertheless, the lack of information still points to the paucity of information regarding African-American men as they manage their type-2 diabetes.

The participation and inclusion of African-African men in public health research studies is imperative for addressing health disparities among this population. Nonetheless, for many different reasons, participation is low in many research studies. ${ }^{[32]}$ Distrust of researchers by many African Americans poses a well-documented, serious challenge to investigator efforts to meet the mandates that require inclusion of minorities in research set out by the National Institutes of Health (NIH), the federal agency in the US that funds a significant amount of biomedical and public health research and sets the national research agenda. Gamble (1997) asserts that the history of medical experimentation on AfricanAmericans during slavery laid the foundation of distrust. ${ }^{[20]}$

Historically, nonparticipation of African-Americans in research has been linked to the history of racism in medical research. ${ }^{[20,24,33-37]}$ The most powerful example of this is the Tuskegee Syphilis Study. For many black men, the Tuskegee study became a symbol of their mistreatment by the medical establishment, a metaphor for deceit, conspiracy, malpractice, and neglect, if not outright racial genocide. ${ }^{[38]}$ In regards to recruitment, researchers would do well to solicit and incorporate the suggestions of AfricanAmerican community members and potential participants in designing research protocols and recruitment strategies. The model of community consent and a collaborative relationship with the population under investigation is not new, and its use has been described in the United States ${ }^{[8,39-41]}$ as well as international communities. ${ }^{[42]}$ The challenge that this poses to researchers however, are finding ways to adequately implement community consent in the African-American population in which the collective community can be valued as highly as the individual.

Notable examples of studies of this type include those published by Hammond and colleagues, ${ }^{[25,43,44]}$ in terms of sample size and representation of African-American men and the approaches used to recruit and retain their participants. Hammond has the largest sample of AfricanAmerican men utilized in studies of this type, yet their work is not specific to type-2 diabetes. The work of Hammond and colleagues is to be commended given their unparalleled ability to recruit, retain, and follow hundreds of African-American men over time, and consequentially making meaningful contributions to the extant literature regarding African-American men and chronic disease. The problem for the remainder of the behavioral science and health promotion world is our inability to replicate and enhance what Hammond's team has accomplished. Until there is a sizeable cadre of researchers who are able to achieve similar success, progress toward meaningful new knowledge of type-2 will not be achieved.

\section{Limitations}

Implications of this study are that it offers a comprehensive view of various points of concentration for how often African-American men are included in research, and consequentially identifies gaps in the knowledge base in this area. Despite its usefulness, the review has several constraints that should be considered. It is possible articles were missed due to search strategies employed, or overlooked in the identification and screening process, which may cause the conclusions based upon the final criteria may be incorrect. We assert, however, that it is unlikely as we utilized the assistance of a medical reference librarian, with expertise in systematic literature searches and reviews, to validate our search protocol and findings. Another limitation is that this review focused on published 
studies written in English. It is possible that studies about African-American men have been published in a language other than English, but we worked under the assumption that the likelihood was negligible. Third, this search was centered on African-American men in the United States, who are living with type 2 diabetes and excluded those with other chronic diseases. We believe this restriction is appropriate given the aims of the study. The search for publications was restricted to papers available between 1996 and 2012. Consequently, it is possible that papers published prior to 1996 may be missed. However, our preliminary investigation revealed very few publications related to diabetes self-management prior to 1996. This was due largely to the inability to easily self-monitor hemoglobin A1c levels until the mass availability of glucose monitoring strips in 1996.

Despite potential limitations, the review provides insight on guidance and direction for future research, identification of the difficulty of recruiting and barriers to participation in empirical research, and the need for developing culturally appropriate, effective recruitment strategies. These strategies should firmly address factors such as lack of minority researchers, socioeconomic status, physician reassurance, factors being studied, mistrust, and data confidentiality.

\section{Implications for research and practice}

Increasing the number of African-American male participants in empirical research requires an improved understanding of the factors affecting the decision to participate. Attention to sensitivity of information collected and collaboration with African-American investigators, colleges, universities, the medical sector, community members, and researchers may improve African-American male representation in empirical research studies. Also, strategies employed by Hammond and colleagues, and others like them should be replicated. Therefore, future research efforts should proactively address recruitment methods tailored to AfricanAmerican men. Investigators would do well to solicit and incorporate the suggestions of African-American community members and potential participants in designing research protocols and recruitment strategies. ${ }^{[16]}$ The model of community consent and a collaborative relationship with the population under investigation is not new, and its use has been described in the United States ${ }^{[8,39-41]}$ as well as international communities. ${ }^{[42]}$ However, finding ways to effectively implement community consent, as a complement to individual consent, may be particularly important in AfricanAmerican and other ethnic minority populations in which the collective community can be valued as highly as the individual.

Not only might this inclusive approach lead to fewer failed efforts, it could help forge strong community partnerships; thereby, transcending the devastating effects of societal mistrust. Researchers should encourage open discourse on the past misuse of minority participants that generated the overall distrust of researchers and describe provisions that they have made to protect participants in their particular studies. The presence of institutional review boards has done little to alleviate fear and suspicion of research among racial/ethnic minorities; ${ }^{[45,46]}$ therefore, acknowledging institutional review board approval for a project is not sufficient. Researchers should also provide frank explanations for studies and initiatives that specifically target racial/ethnic minorities or that are likely to result in the disproportionate representation of racial/ ethnic minorities among study participants.

\section{REFERENCES}

1. Shavers-Hornaday VL, Lynch CF, Burmeister LF, Torner JC. Why are African-Americans under-represented in medical research studies? Impediments to participation. Ethn Health 1997;2:31-45.

2. Areán PA, Gallagher-Thompson D. Issues and recommendations for the recruitment and retention of older ethnic minority adults into clinical research. J Consult Clin Psychol 1996;64:875-80.

3. Hodge FS, Weinmann S, Roubideaux Y. Recruitment of American Indians and Alaska Natives into clinical trials. Ann Epidemiol 2000;10:S41-8.

4. McCabe MS, Varricchio CG, Padberg RM. Efforts to recruit the economically disadvantaged to national clinical trials. Semin Oncol Nurs 1994;10:123-9.

5. Stark N, Paskett E, Bell R, Cooper MR, Walker E, Wilson $A$, et al. Increasing participation of minorities in cancer clinical trials: Summary of the "Moving Beyond the Barriers" Conference in North Carolina. J Natl Med Assoc 2002;94:31-9.

6. Zambrana RE, Carter-Pokras O. Health data issues for Hispanics: Implications forpublic health research. J Health Care Poor Underserved 2001;12:20-34.

7. Cook LS, de Mange BP. Gaining access to Native American cultures by non-Native American nursing researchers. Nurs Forum 1995;30:5-10.

8. Kaluzny A, Brawley O, Garson-Angert D, Shaw J, Godley P, Warnecke R, et al. Assuring access to state-of-the-art care for U. S. minority populations: The first 2 years of the Minority-Based Community Clinical Oncology Program. J Natl Cancer Inst 1993;85:1945-50.

9. Dennis BP, Neese JB. Recruitment and retention of AfricanAmerican elders into community-based research: Lessons learned. Arch Psychiatr Nurs 2000;14:3-11.

10. Harris Y, Gorelick PB, Samuels P, Bempong I. Why African-Americans may not be participating in clinical trials. J Natl Med Assoc 1996;88:630-4.

11. Powell JH, Fleming Y. Making medicines for America: The case for clinical trial diversity. J Natl Med Assoc 2000;92:507-14. 
12. Moyé LA, Powell JH. Evaluation of ethnic minorities and gender effects in clinical trials: Opportunities lost and rediscovered. J Natl Med Assoc 2001;93:29-34S.

13. Ness RB, Nelson DB, Kumanyika SK, Grisso JA. Evaluating minority recruitment into clinical studies: How good are the data? Ann Epidemiol 1997;7:472-8.

14. Baker R. Minority distrust of medicine: A historical perspective. Mt Sinai J Med 1999;66:212-22.

15. Blendon RJ, Scheck AC, Donelan K, Hill CA, Smith M, Beatrice D, et al. How white and African-Americans view their health and social problems. Different experiences, different expectations. JAMA 1995;273:341-6.

16. Corbie-Smith G, Thomas SB, St. George DM. Distrust, race, and research. Arch Intern Med 2002;162:2458-63.

17. Davis SM, Reid R. Practicing participatory research in American Indian communities. Am J Clin Nutr 1999;69:755-9S.

18. Earl CE, Penney PJ. The significance of trust in the research consent process with African-Americans. West $\mathrm{J}$ Nurs Res 2001;23:753-62.

19. Fouad MN, Partridge E, Green BL, Kohler C, Wynn T, Nagy $\mathrm{S}$, et al. Minority recruitment in clinical trials: A conference at Tuskegee, researchers and the community. Ann Epidemiol 2000;10:S35-40.

20. Gamble VN. Under the shadow of Tuskegee: African-Americans and health care. Am J Public Health 1997;87:1773-8.

21. LaVeist TA, Nickerson, KJ, Bowie JV. Attitudes about racism, medical mistrust, and satisfaction with care among African-American and white cardiac patients. Med Care Res Rev 2000;57:146-61.

22. McGary H. Distrust, social justice, and health care. Mt Sinai J Med 1999;66:236-40.

23. Sambo BH. Strong Heart Study Investigators. The Strong Heart Study: Interaction with and benefit to American Indian communities. Am J Med Sci 2001;322:282-5.

24. Thomas SB, Quinn SC. The Tuskegee Syphilis Study, 1932 to 1972: Implicationsfor HIV education and AIDS risk education programs in the Black community. Am J Public Health 1991;81:1498-505.

25. Hammond WP. Psychosocial correlates of medical mistrust among African American men. Am J Community Psychol 2010;45:87-106.

26. Mansfield AK, Addis ME, Courtenay W. Measurement of men's help-seeking: Development and evaluation of the barriers to help seeking scale. Psychol Men Masculin 2005;6:95-108.

27. Hammond WP, Mattis JS. Being a man about it: Manhood meaning among African American men. Psychol Men Masculin 2005;6:114-26.

28. LaVeist TA, Isaac LA, Williams KP. Mistrust of health care organizations isassociated with underutilization of health services. Health Serv Res 2009;44:2093-105.

29. Lillie-Blanton M, Brodie M, Rowland D, Altman D, Mclntosh M. Race, ethnicity, and the healthcare system: Public perceptions and experiences. Med Care Res Rev 2000;57:218-35.

30. Hendricks LE, Hendricks RT. The effect of diabetes self-management education with frequent follow-up on the health outcomes of AfricanAmerican Men. Diabetes Educ 2000;26:995-1002.
31. Garrard JG. Health sciences literature review made easy: The matrix method. Edited by Sullivan K. $2^{\text {nd }}$ ed. Boston, MA: Jones and Bartlett; 2004; p. 30-3.

32. Freimuth VS, Quinn SC, Thomas SB, Cole G, Zook E, Duncan T. African Americans' views on research and the Tuskegee Syphilis Study. Soc Sci Med 2001;52:797-808.

33. Swanson GM, Ward AJ. Recruiting minorities into clinical trials: Toward a participant-friendly system. J Natl Cancer Inst 1995;87:1747-59.

34. Brandt AM. Racism and research: The case of the Tuskegee Syphilis Study. Hastings Cent Rep 1978;8:21-9.

35. Caplan AL. Twenty years after. The legacy of the Tuskegee Syphilis Study. When evil intrudes. Hastings Cent Rep 1992;22:29-32.

36. Edgar $\mathrm{H}$. Twenty years after. The legacy of the Tuskegee Syphilis Study. Outside the community. Hastings Cent Rep 1992;22:32-5.

37. King PA. Twenty years after. The legacy of the Tuskegee Syphilis Study. The dangers of difference. Hastings Cent Rep 1992;22:35-8.

38. Jones JH. Bad blood. Simon and Schuster. New York, NY; 1993; p. 206-9.

39. Foster MW, Bernsten D, Carter TH. A model agreement for genetic research insocially identifiable populations. Am J Hum Gen 1998;63:696-702.

40. Freeman HP. The impact of clinical trial protocols on patient care systems in a large city hospital. Access for the socially disadvantaged. Cancer 1993;72:2834-8.

41. Gorelick PB, Richardson D, Hudson E, Perry C, Robinson D, Brown N, et al. Establishing a community network for recruitment of African Americans into a clinical trial. The African-American antiplatelet stroke Prevention Study (AAASPS) experience. J Natl Med Assoc 1996;88:701-4.

42. Barry, M. Ethical considerations of human investigation in developing countries: The AIDS dilemma. N Engl J Med 1988;319:1083-6.

43. Hammond WP, Matthews D, Corbie-Smith G. Psychosocial factors associated with routine health examination scheduling and receipt among African-American men. J Natl Med Assoc 2010;102:276-89.

44. Hammond WP, Matthews D, Mohottige D, Agyemang A, CorbieSmith G. Masculinity, medical mistrust, and preventive health services delays among community-dwelling African-American men. J Gen Intern Med 2010;25:1300-8.

45. Mouton CP, Harris S, Rovi S, Solorzano P, Johnson MS. Barriers to black women's participation in cancer clinical trials. J Natl Med Assoc 1997;89:721-7.

46. Byman B. Out from the shadow of Tuskegee. Fighting racism in medicine. Minn Med 1991;74:15-20.

How to cite this article: Sherman LD, J McKyer E. Where are they? The inclusion of African-American men in empirical studies of type 2 diabetes self-care management. J Soc Health Diabetes 2015;3:102-14.

Source of Support: This study was funded, in part, by the Transdisciplinary Center for Health Equity Research (TCHER). TCHER is housed in the Department of Health and Kinesiology, College of Education and Human Development, at Texas A and M University. I gratefully acknowledge the study participants who gave of their time and showed me patience throughout the process. Conflict of Interest: None declared. 